\title{
Synthesis and evaluation of cytotoxic activities of some substituted isoxazolone derivatives
}

\author{
Sevim Rollas ${ }^{1}$, Şila Kokyan¹, Bedia Koçyiğit-Kaymakçıŏlu1', Suna Özbaş-Turan², \\ Jülide Akbuğa²
}

\begin{abstract}
Several isoxazolone derivatives were synthesized, starting from substituted 1,3,4-thiadiazoles and 1,2,4-triazole-3-thione. In the first part of the research, compounds 2-(4-aminophenyl)-5-alkyl/arylamino-1,3,4-thiadiazoles (4a-e) and 5-(4-aminophenyl)-4-substitude-2,4-dihydro-3H-1,2,4-triazole-3-thiones (5a-c) were prepared from ethyl 4-aminobenzoate. In the second part, compounds, which were prepared by coupling the diazonium salts of aromatic primary amines with ethyl acetoacetat (6a-e, 7a-c) were cyclized with hydroxylamine hydrochloride and sodium acetate in ethanol and yielded 3-methyl-4-[2-\{4-[5 alkyl/ arylamino)-1,3,4-thiadiazol-2-yl]phenyl\} hydrazinylidene]isoxazol-5(4H)-one (8a-e) 3-methyl-4-[2-\{4-[4-(4-alkyl/aryl)-5-thioxo-4,5-dihydro-1H-1,2,4-triazol-3-yl]phenyl\}hydrazinylidene]isoxazol-5(4H)-one $(8 \mathrm{f}-\mathrm{g})$. The structures of the synthesized compounds were confirmed by elemental analysis (C,H,N,S), UV, IR, 1H-NMR and mass spectroscopic methods. Cytotoxicity of these compounds were evaluated by using HEK293 cell line of MTT [3-(4,5-dimethylthiazole-2-yl)-2,5-diphenyltetrazolium bromide] assay. The highest inhibitions were confirmed as \%45.72 for the compound 3-methyl-4-[2-(4-\{5-[(4-methoxyphenyl) amino]-1,3,4-thiadiazol-2-yl\}phenyl)hydrazinylidene]isoxazol-5(4H)-one (8e) and \%33.07 for the compound 3-methyl-4-[2-(4-\{5-[(4-methylphenyl)amino]-1,3,4-thiadiazol-2-yl\}phenyl)hydrazinylidene]isoxazol-5(4H)-one (8a).
\end{abstract}

KEYWORDS: 1,2,4-Triazole-3-thione, 1,3,4-thiadiazole, isoxazolone, hydrazone, cytotoxic activity

\section{INTRODUCTION}

Isoxazoles have a long history of application in pharmaceutical and agrochemical industry (1). Isoxazole-5-one derivatives are also known for their biological activity as anti-androgenic, antitubercular, cytotoxic and antibacterial agents (2-6).

The recognition of the pharmacological activity of some isoxazole derivatives such as Gantricin, a sulfa drug from amino isoxazole and cycloserine a simple derivative of 3-isoxazolidone as antibiotics has aroused a new interest in this field (7). Many synthesis methods are reported about the cyclization of isoxazolones derivatives. One of the most useful method to obtain isoxazolones relies on the cyclocondensation of $\beta$-keto esteres or $\alpha, \beta$ unsaturated esters with hydrazines or hydroxylamines, respectively (8-11). In the present study, isoxazole derivatives have been synthesized by the reaction of 1,3-dicarbonyl compounds with hydroxylamines in basic medium (12-13). On the other hand, it has been reported that 1,2,4-triazole derivatives possess a wide spectrum of chemotherapeutic activities including anti-inflammatory, anticancer, antidepressant, antibacterial, anticonvulsant and as well as antifungal properties (14-22). In fact, some of their derivatives are active constituents of currently used drugs such as Fluconazole and Itraconazole as potent antifungal agents (23). Furthermore, various 1,3,4-thiadiazole derivatives were shown to possess excellent pharmacological properties such as antimicrobial, analgesic and anti-inflammatory activities (24-27). On the basis of these observations, we aimed to synthesize a new class of heterocyclics, wherein potent
AFFILIATIONS

1 M.Ü. Eczacılık Fak.,

Farmasötik Kimya, İstanbul,

Türkiye

2M.Ü.Eczacılık Fak.

Farmasötik Biyoteknoloji,

İstanbul, Türkiye

CORRESPONDENCE

Sevim Rollas

E-mail:

sevim@sevimrollas.com

Received:

31.03.2011

Revision:

26.05.2011

Accepted:

26.05.2011 
1,2,4-triazole and 1,3,4-thiadizaole moiety is attached to position 4 of the isoxazolone ring. Therefore, a series of new 3-methyl-4-(2-\{4-[5 alkyl/arylamino )-1,3,4-thiadiazol-2-yl]phenyl\} hydrazinylidene)isoxazol-5(4H)-one and 3-methyl-4-(2-\{4-[4(4-aryl)-5-thioxo-4,5-dihydro-1H-1,2,4-triazol-3-yl]phenyl hydrazinylidene) isoxazol-5(4H)-one were synthesized and their structures were confirmed by means of UV, IR, ${ }^{1} \mathrm{H}-\mathrm{NMR}$, mass spectroscopy and elemental analysis. All the synthesized compounds were screened for their cytotoxic activities by using HEK293 cell line of MTT [3-(4,5-dimethylthiazole-2-yl)-2,5diphenyltetrazolium bromide] Assay.

\section{EXPERIMENTAL}

\subsection{Chemistry}

All chemicals and solvents were purchased from Merck, Aldrich, or Fluka. Melting points were determined by a "Schmelzpunktbestimmer" SMP II and were uncorrected. The reactions were monitored on Merck pre-coated aluminium TLC plates 60F-254 and the products were visualized by UV-light using chloroform and acetone $(50: 50 \mathrm{v} / \mathrm{v})$ as solvent system. The UV spectra were recorded on a Schimadzu UV-1601 spectrometry. The IR spectra were recorded on a Schimadzu FTIR 84005 spectrometry. ${ }^{1} \mathrm{H}-\mathrm{NMR}$ spectra were recorded in DMSO on a Bruker Avance-DPX-400 spectrometer in DMSO- $\mathrm{d}_{6}$ and chemical shifts were given in $\delta$ ppm with tetramethylsilane. The splitting patterns of ${ }^{1} \mathrm{H}-\mathrm{NMR}$ were designed as follows: s: singlet, $\mathrm{d}$ : doublet, t: triplet, q: quartet, m: multiplet. Mass spectrometry was performed using an Agilent 1100 MSD spectrometer. Elemental analysis was performed using a Vario MICROV1.5.7

Synthesis of ethyl 4-(benzoylamino)benzoate (1). Benzocaine $(0.03 \mathrm{~mol})$ was dissolved in $36 \mathrm{~mL}$ ether. Benzoylchloride (3.6 $\mathrm{mL}$ diluted in $3.6 \mathrm{~mL}$ ether.) was added drop by drop and stirred, after evaporating ether, the solid product was washed with distilled water until the smell of benzoyl chloride disappeared and then crystallized from ethanol.

Synthesis of 4-(benzoylamino)benzoylhydrazine (2). $9 \mathrm{~mL}$ hydrazine hydrate was added to ethyl 4-(benzoylamino)benzoate (28). The mixture was refluxed at $110-130^{\circ} \mathrm{C}$ for $30 \mathrm{~min}$ utes. After adding $15 \mathrm{~mL}$ ethanol, the mixture was heated in water bath for 45 minutes. The residue was filtered, washed with water and crystallized from ethanol.

General procedure for the preparation of 1-[4-(benzoylamino)benzoyl]-4-alkyl/-arylthiosemicarbazide (3a-h). $0.005 \mathrm{~mol}$ alkyl/arylisothiocyanate was added to the solution of 0.005 mol 4-(benzoylamino)benzoylhydrazine in $40 \mathrm{~mL}$ ethanol. The mixture was refluxed on water bath for $2-2.5 \mathrm{~h}$. The solid product, obtained on cooling, was washed with distilled water and crystallized from ethanol.

General procedure for the preparation of 2-(4-aminophenyl)5-alkyl/arylamino-1,3,4-thiadiazole (4a-e). $15-20 \mathrm{~mL} \quad 50 \%$ $\mathrm{H}_{2} \mathrm{SO}_{4}$ was added to $3 \mathrm{a}-\mathrm{f}(0.006 \mathrm{~mol})$ and refluxed at $110-150^{\circ} \mathrm{C}$ for $5 \mathrm{~h}$. The mixture was neutralized with $2 \mathrm{~N} \mathrm{NaOH}$. The precipitate was filtered, washed with water and crystallized from ethanol (28).

General procedure for the preparation of 5-(4-aminophenyl)4-alkyl/aryl-1,2,4-triazole-3-thione (5a-c). 15-20 mL 2N NaOH was added to $3 a-f(0.005 \mathrm{~mol})$ and refluxed on water bath for 4 h. The reaction mixture was neutralized with $10 \% \mathrm{HCl}$. The precipitate was filtered, washed with water and crystallized from ethanol (28).
General procedure for the preparation of coupling products (6a-e, 7a-c).

$1 \mathrm{ml}$ of an ice-cold solution of sodium nitrite $(10 \%)$ containing $0.2 \mathrm{ml}$ hydrochloric acid (37\%) is added drop by drop to 0.001 mol 2-(4-aminophenyl)-5-alkyl/arylamino-1,3,4-thiadiazolles or 5-(4-aminophenyl)-4-alkyl/arylamino-1,2,4-triazole-3thiones. The reaction mixture is then poured into a mixture of $0.001 \mathrm{~mol}$ ethylacetoacetat and $5 \mathrm{~g}$ sodium acetate in ethanol (5 $\mathrm{mL}$ ) by vigorous stirring. The precipitate was filtered, washed with water, dried and crystallized from appropriate solvent (28).

General procedure for the preparation of 5-isoxazolone derivatives (8a-g). $0.001 \mathrm{~mol}$ coupling product, $0.001 \mathrm{~mol}$ hydroxylamine hydrocloride, $0.5 \mathrm{~g}$ sodium acetate were solved in $5 \mathrm{~mL}$ ethanol and refluxed on water bath for 3-3.5 h. The precipitate was filtered, washed with water and crystallized from ethanol.

3-methyl-4-[2-(4-\{5-[(4-methylphenyl)amino]-1,3,4-thiadiazol2-yl\}phenyl)hydrazinylidene]isoxazol-5(4H)-one (8a): Yield $69 \%, \mathrm{mp} 240-242{ }^{\circ} \mathrm{C}$. UV $\lambda_{\max }(\mathrm{EtOH})(\mathrm{nm}): 415.5,327.5,249.5$. IR ( $\left.\overline{\mathrm{v}}_{\max }, \mathrm{cm}^{-1}\right)$ : $3194(\mathrm{~N}-\mathrm{H}) ; 1716(\mathrm{C}=\mathrm{O}) ; 1553,1516(\mathrm{C}=\mathrm{N}) .{ }^{1} \mathrm{H}$ NMR (400 MHz) (DMSO-d $\left.d_{6} / \mathrm{TMS}\right) \delta$ ppm: $2.23\left(\mathrm{~s}, 3 \mathrm{H},-\mathrm{CH}_{3}\right)$; $2.30\left(\mathrm{~s}, 3 \mathrm{H},-\mathrm{CH}_{3}\right) ; 7.20(2 \mathrm{H}, \mathrm{d}, \mathrm{J}: 8 \mathrm{~Hz}, \mathrm{Ar}-\mathrm{H}) ; 7.51(2 \mathrm{H}, \mathrm{d}, \mathrm{J}: 8$ $\mathrm{Hz}, \mathrm{Ar}-\mathrm{H}) ; 7.93$ (2H, d, J: $9 \mathrm{~Hz}, \mathrm{Ar}-\mathrm{H}) ; 8.0$ (2H, d, J: $9 \mathrm{~Hz}, \mathrm{Ar}-\mathrm{H})$; $10.52(\mathrm{~s}, 1 \mathrm{H},-\mathrm{NH}) ; 12.91(\mathrm{~s}, 1 \mathrm{H},=\mathrm{C}-\mathrm{N}=\underline{\mathrm{NH}})$. Anal. Calcd. for $\mathrm{C}_{19} \mathrm{H}_{16} \mathrm{~N}_{6} \mathrm{O}_{2} \mathrm{~S} ; \mathrm{C}, 58.15 ; \mathrm{H}, 4.11 ; \mathrm{N}, 21.42 ; \mathrm{S}, 8.15$ Found: C, 57.74; H, 4.12; N, 20.71; S, 7.90.

3-methyl-4-[2-\{4-[5-(methylamino)-1,3,4-thiadiazol-2-yl] phenyl hhydrazinylidene]isoxazol-5(4H)-one (8b): ): Yield 80 $\%, \mathrm{mp} 209-211{ }^{\circ} \mathrm{C}$. UV $\lambda_{\max }(\mathrm{EtOH})(\mathrm{nm}): 421.5,304,220$. IR $\left(\overline{\mathrm{v}}_{\max }, \mathrm{cm}^{-1}\right)$ : $3207(\mathrm{~N}-\mathrm{H}) ; 1716(\mathrm{C}=\mathrm{O}) ; 1556(\mathrm{C}=\mathrm{N}) .{ }^{1} \mathrm{H}$ NMR (400 MHz), (DMSO-d $d_{6}$ TMS) $\delta$ ppm: 2.27 (s, 3H, $\left.-\mathrm{CH}_{3}\right) ; 2.95$ (s, $\left.3 \mathrm{H},-\mathrm{CH}_{3}\right) ; 7.83(2 \mathrm{H}, \mathrm{d}, \mathrm{J}: 9 \mathrm{~Hz}, \mathrm{Ar}-\mathrm{H}) ; 7.85(2 \mathrm{H}, \mathrm{d}, \mathrm{J}: 9 \mathrm{~Hz}, \mathrm{Ar}-$ $\mathrm{H}), 8.19$ (s, 1H, -NH-); 12.82 (s, 1H, =C-N=NH-). Anal. Calcd. for $\mathrm{C}_{13} \mathrm{H}_{12} \mathrm{~N}_{6} \mathrm{O}_{2} \mathrm{~S} .1 \mathrm{H}_{2} \mathrm{O} ; \mathrm{C}, 46.6 ; \mathrm{H}, 4.2 ; \mathrm{N}, 25.1 ; \mathrm{S}, 9.5$ Found: C, $45.7 ; \mathrm{H}, 4.1 ; \mathrm{N}, 24.2 ; \mathrm{S}, 9.2$.

3-methyl-4-[ 2-\{4-[5-(propylamino)-1,3,4-thiadiazol-2-yl]phenyl\}hydrazinylidene]isoxazol-5(4H)-one

(8c): ): Yield $64 \%, \mathrm{mp} 214{ }^{\circ} \mathrm{C}$. UV $\lambda_{\max }(\mathrm{EtOH})(\mathrm{nm}): 423.5$, 302.5. IR ( $\left.\overline{\mathrm{v}}_{\max }, \mathrm{cm}^{-1}\right)$ : $3200(\mathrm{~N}-\mathrm{H}) ; 1720(\mathrm{C}=\mathrm{O}) ; 1552(\mathrm{C}=\mathrm{N}) \cdot{ }^{1} \mathrm{H}$ NMR (400 MHz), (DMSO-d $\left.{ }_{6} / \mathrm{TMS}\right) \delta$ ppm: $0.92\left(\mathrm{t}, 3 \mathrm{H}, \mathrm{CH}_{3}\right)$; $1.57\left(\mathrm{~m}, 2 \mathrm{H}, \mathrm{CH}_{2}\right) ; 2.27\left(\mathrm{~s}, 3 \mathrm{H},-\mathrm{CH}_{3}\right) ; 3.26\left(\mathrm{t}, 2 \mathrm{H},-\mathrm{CH}_{2}\right) ; 7.74$ $(2 \mathrm{H}, \mathrm{d}, \mathrm{J}: 9 \mathrm{~Hz}, \mathrm{Ar}-\mathrm{H}), 7.81(2 \mathrm{H}, \mathrm{d}, \mathrm{J}: 9 \mathrm{~Hz}, \mathrm{Ar}-\mathrm{H}), 7.98$ (s, 1H, -NH-), 12.82 (s, 1H, =C-N=NH-). CI-MS, m/z (\%): $345\left(\mathrm{M}^{++1}\right.$, 100). Anal. Calcd. For $\mathrm{C}_{15} \mathrm{H}_{16} \mathrm{~N}_{6} \mathrm{O}_{2} \mathrm{~S}$ : C, 52.3; H, 4.7; N, 24.4; , 9.3 Found: C, 51.9; H, 4.6; N, 24.1; S, 9.0.

3-methyl-4-[2-(4-\{5-[(4-ethylphenyl)amino]-1,3,4-thiadiazol-2yl\}phenyl)hydrazinylidene]isoxazol-5(4H)-one (8d): Yield $60 \%$, mp 231-233 oC. UV $\lambda_{\max .}(\mathrm{EtOH})(\mathrm{nm}): 414,321,251$. IR $\left(\overline{\mathrm{v}}_{\max }, \mathrm{cm}^{-1}\right): 3192(\mathrm{~N}-\mathrm{H}) ; 1716(\mathrm{C}=\mathrm{O}) ; 1548,1506(\mathrm{C}=\mathrm{N}) .{ }^{1} \mathrm{H}$ NMR (400 MHz), (DMSO-d 6 /TMS) $\delta$ ppm: $1.16\left(\mathrm{t}, 3 \mathrm{H},-\mathrm{CH}_{3}\right)$; $2.28\left(\mathrm{~s}, 3 \mathrm{H},-\mathrm{CH}_{3}\right) ; 2.55\left(\mathrm{~m}, 2 \mathrm{H},-\mathrm{CH}_{2}\right) ; 7.19(2 \mathrm{H}, \mathrm{d}, \mathrm{J}: 8 \mathrm{~Hz}, \mathrm{Ar}-$ $\mathrm{H}) ; 7.54$ (2H, d, J: $8 \mathrm{~Hz}, \mathrm{Ar}-\mathrm{H}) ; 7.71$ (2H, d, J: 9 Hz, Ar-H); 7.91 $(2 \mathrm{H}, \mathrm{d}, \mathrm{J}: 9 \mathrm{~Hz}, \mathrm{Ar}-\mathrm{H}) ; 10.49$ (s, 1H, -NH); 12.83 (s, 1H, =C$\mathrm{N}=\mathrm{NH}-$ ). Anal. Calcd. For $\mathrm{C}_{20} \mathrm{H}_{18} \mathrm{~N}_{6} \mathrm{O}_{2} \mathrm{~S} .1 \mathrm{H}_{2} \mathrm{O}: \mathrm{C}, 56.5 ; \mathrm{H}, 4.7$; N, 19.7; S, 7.5 Found: C, 56.9; H, 4.4; N, 19.0; S, 7.6.

3-methyl-4-[2-(4-\{5-[(4-methoxyphenyl)amino]-1,3,4-thiadiazol-2-yl\}phenyl)hydrazinylidene] isoxazol-5(4H)-one (8e): Yield 50 \%, mp 229-231 ${ }^{\circ} \mathrm{C}$. UV $\lambda_{\max }(\mathrm{EtOH})(\mathrm{nm}): 410.0,249.5$ 
251. IR ( $\left.\overline{\mathrm{v}}_{\max }, \mathrm{cm}^{-1}\right): 3190(\mathrm{~N}-\mathrm{H}) ; 1716(\mathrm{C}=\mathrm{O}) ; 1549,1506(\mathrm{C}=\mathrm{N})$. ${ }^{1} \mathrm{H}-\mathrm{NMR}(400 \mathrm{MHz}),\left(\mathrm{DMSO}-\mathrm{d}_{6} / \mathrm{TMS}\right) \delta \mathrm{ppm}: 2.28(\mathrm{~s}, 3 \mathrm{H}$, $\left.-\mathrm{CH}_{3}\right) ; 3.83\left(\mathrm{~s}, 3 \mathrm{H},-\mathrm{OCH}_{3}\right) ; 6.85(2 \mathrm{H}, \mathrm{d}, \mathrm{J}: 9 \mathrm{~Hz}, \mathrm{Ar}-\mathrm{H}) ; 7.05(2 \mathrm{H}$, d, J: $9 \mathrm{~Hz}, \mathrm{Ar}-\mathrm{H}) ; 7.57$ (2H, d, J: $8 \mathrm{~Hz}, \operatorname{Ar}-\mathrm{H}) ; 7.79$ (d, J: $8 \mathrm{~Hz}$, $2 \mathrm{H}, \mathrm{Ar}-\mathrm{H}) ; 10.22$ (s, $1 \mathrm{H},-\mathrm{NH}) ; 10.36$ (s, $\left.1 / 4 \mathrm{H}_{2} \mathrm{SO}_{4}-\mathrm{OH}\right) 12.83$ (s, 1H, =C-N=NH-). CI-MS, m/z (\%): $409.0\left(\mathrm{M}^{+}+1,20.2\right)$. Anal. Calcd. For $\mathrm{C}_{19} \mathrm{H}_{16} \mathrm{~N}_{6} \mathrm{O}_{3} \mathrm{~S} .1 / 4 \mathrm{H}_{2} \mathrm{SO}_{4}$ : C, 52.60; H, 3.81; N, 19.30; S, 9.24. Found: C, 52.20; H, 3.56; N, 18.18; S, 9.26.

3-methyl-4-\{2-[4-(4-ethyl-5-thioxo-4,5-dihydro-1H-1,2,4-triazol3-yl)phenyl]hydrazinylidene\} isoxazol-5(4H)-one (8f): Yield 59 $\%$, mp $222^{\circ} \mathrm{C}$; Lit. mp $220^{\circ} \mathrm{C}$ (13). ${ }^{1} \mathrm{H}-\mathrm{NMR}$ (400 MHz), (DMSO$\left.\mathrm{d}_{6} / \mathrm{TMS}\right) \delta$ ppm: $1.05\left(\mathrm{t}, 3 \mathrm{H}, \mathrm{CH}_{2}-\mathrm{CH}_{3}\right) ; 2.27\left(\mathrm{~s}, 3 \mathrm{H},-\mathrm{CH}_{3}\right) ; 4.04$ $\left(\mathrm{m}, 2 \mathrm{H},-\mathrm{CH}_{2}\right) ; 7.73$ (d, 2H, J. $\left.9 \mathrm{~Hz}, \mathrm{Ar}-\mathrm{H}\right), 7.80$ (d, J: $9 \mathrm{~Hz}, 2 \mathrm{H}$, Ar-H); 12.81 (s, 1H, =C-N=H- $)$; 13.95 (s, 1H, -NH-).

3-methyl-4-(2-\{4-[4-(4-methoxyphenyl)-5-thioxo-4,5-dihydro$1 H$-1,2,4-triazol-3-yl]phenyl\}hydra-zinylidene)isoxazol-5(4H)one (8g): Yield $63 \%$, mp 230-232 ${ }^{\circ} \mathrm{C}$. UV $\lambda_{\max }(\mathrm{EtOH})(\mathrm{nm})$ : 399.0, 259.0, 231.0. IR ( $\left.\overline{\mathrm{v}}_{\max } \mathrm{cm}^{-1}\right)$ : $3031(\mathrm{~N}-\mathrm{H}) ; 1716(\mathrm{C}=\mathrm{O})$; 1553, $1520(\mathrm{C}=\mathrm{N}) .{ }^{1} \mathrm{H}-\mathrm{NMR}$ (400 MHz), (DMSO-d $\left.6 / \mathrm{TMS}\right)$ $\delta$ ppm: $2.31\left(\mathrm{~s}, 3 \mathrm{H},-\mathrm{CH}_{3}\right) ; 3.78\left(\mathrm{~s}, 3 \mathrm{H},-\mathrm{OCH}_{3}\right) ; 7.03$ (d, J: $9 \mathrm{~Hz}$, 2H, Ar-H); 7.27 (dd, J: 9 Hz, 4H, Ar-H); 7.61 (d, J: 9 Hz, 2H, Ar$\mathrm{H}) ; 12.72$ (s, 1H, =C-N=NH-); 14.09 (s, 1H, -NH). CI-MS, m/z (\%): $409.0\left(\mathrm{M}^{+}+1,100\right)$. Anal. Calcd. For $\mathrm{C}_{19} \mathrm{H}_{16} \mathrm{~N}_{6} \mathrm{O}_{3} \mathrm{~S} .1 \mathrm{H}_{2} \mathrm{O}$ : C, 53.4; H, 4.2; N, 19.7; S, 7.5 Found: C, 53.8; H, 3.9; N, 18.1; S, 7.6.

\subsection{Cytotoxic activity}

The synthesized compounds were tested for their cytotoxic activities. Cell viability and cytotoxic activity profile of the compounds were analyzed using the MTT assay $(29,30,31)$. MTT is cleaved to formazan by the "succinate-tetrazolium reductase" system (EC 1.3.99.1) which belongs to the mitochondrial respiratory chain and is active only in viable cells.

HEK293 cell line was used for the determination of cytotoxic activity (ATCC $®$ CRL-1573 ${ }^{\mathrm{TM}}$ ). Cytotoxicity testing in vitro was done by the method of modified Woerdenbag et al (30, 31). The MTT metabolic assay was carried out in seeded at the density of $1 \times 10^{4}$ cells/well in 96-well flat-bottom cell culture plates well plates with $200 \mu \mathrm{L}$ of opti-MEM (invitrogen, USA) and incubated for 24 hours at $37^{\circ} \mathrm{C}, 5 \% \mathrm{CO}_{2}$. The following day, media was aspirated and the compounds were dissolved in DMSO and diluted with medium before they were added to the cell cultures at the concentrations of $5 \mu \mathrm{g} /$ $\mathrm{mL}$ and $10 \mu \mathrm{g} / \mathrm{mL}$. Cells were incubated for 48 hrs at $37^{\circ} \mathrm{C}, 5 \% \mathrm{CO}_{2}$. After the incubation period $10 \mu \mathrm{L}$ of the MTT labeling reagent [final concentration $0.5 \mu \mathrm{g} / \mathrm{mL}$ (Cell proliferation kit MTT, Roche, Germany)] was added to each well. The cultures was incubated for 4-12 hours in a humidified atmosphere (e.g. $37^{\circ} \mathrm{C}$, $\left.5.0 \% \mathrm{CO}_{2}\right)$ and $100 \mu \mathrm{L}$ of the solubilization buffer was added into each well. The plate was allowed to stand overnight in the incubator in a humidified atmosphere (e.g. $37^{\circ} \mathrm{C}$, $5.0 \% \mathrm{CO}_{2}$ ), the formazan precipitates were solubilized. Absorbance of the formazan product was measured spectrophotometrically at 550 and $690 \mathrm{~nm}$.

Statistical analyses were done using unpaired Student's t-test using Prism 3.0 (GraphPad Software, San Diego; CA; USA).

\section{RESULTS AND DISCUSSION}

In our research, starting from substituted 1,3,4-thiadiazole and 1,2,4-triazole-3-thione, several isoxazolone derivatives (8a-g) were synthesized. In the first part of this study, compounds 2-(4-aminophenyl)-5-alkyl/arylamino-1,3,4-thiadiazoles (4ae) and 5-(4-aminophenyl)-4-substitude-2,4-dhydro-3H-1,2,4triazole-3-thions (5a-c) were prepared from ethyl 4-aminobenzoate according to the literature [13, 32, 33] (Scheme 1). In the second part, compounds, which were prepared by coupling the diazonium salts of aromatic primary amines with ethyl acetoacetat (6a-e, 7a-c) (6a-e, 7a-c) were cyclized with hydroxylamine hydrochloride and sodium acetate in ethanol and yielded 3-methyl-4-[ 2-\{4-[5-alkyl/arylamino)-1,3,4-thiadiazol-2-yl]phenyl\}hydrazinylidene]isoxazol-5(4H)-one (8a-e) 3-methyl-4-[2-\{4-[4-(4-aryl)-5-thioxo-4,5-dihydro-1H-1,2,4-triazol-3-yl]phenyl\}hydrazinylidene)] isoxazol-5(4H)-one (8f, $\mathbf{g}$ ) (Scheme 2 and 3). Purity of the synthesized isoxazolone compounds (8a-g) were checked by thin layer chromatography.

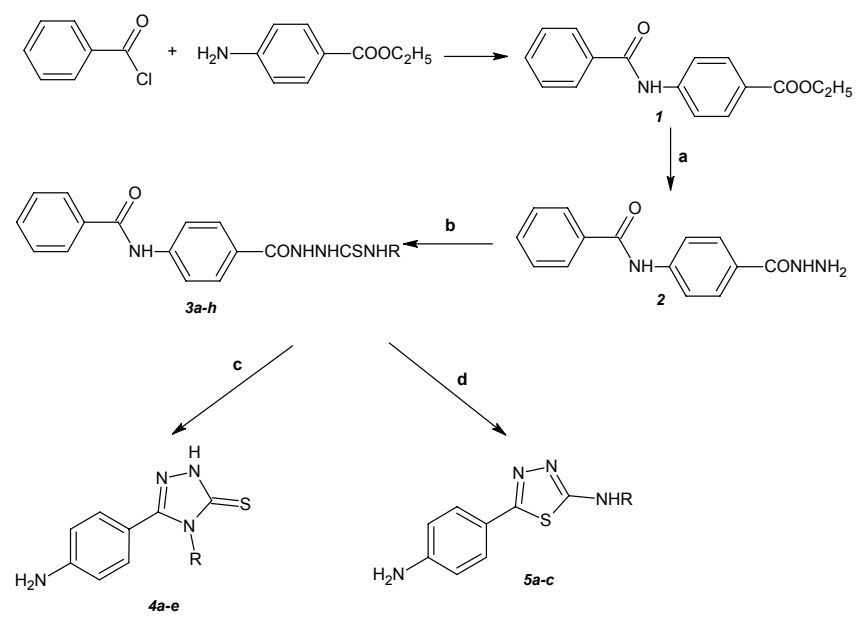

SCHEME 1. Synthesis of intermediate products of 1,3,4-thiadiazole (4a-e) and 1,2,4-triazole-3-thione (5a-c) derivatives

$\mathrm{R}=$ methyl, ethyl, propyl, 4-methylphenyl, 4-methoxyphenyl

Reaction condition: a: $\mathrm{N}_{2} \mathrm{H}_{4} \cdot \mathrm{H}_{2} \mathrm{O}$; b: RNCS; c: $\mathrm{H}_{2} \mathrm{SO}_{4}$, D; d: $\mathrm{NaOH}, \mathrm{D}$.

The structures of new compounds (8a-g) were confirmed by $\mathrm{UV}, \mathrm{IR},{ }^{1} \mathrm{H}-\mathrm{NMR}$, mass spectroscopic (for compound $\mathbf{8 c}$, $8 \mathbf{e}$ and $8 \mathrm{~g}$ ) methods and elemental analysis.

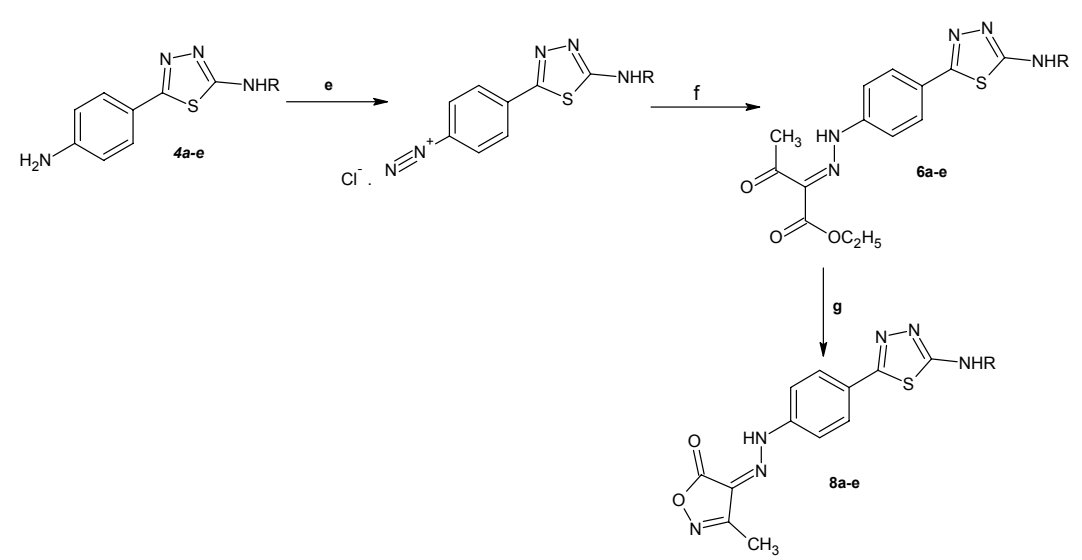

SCHEME 2. Synthesis of 5 -isoxazolone derivatives (8a-e) $\mathrm{R}=$ methyl, ethyl, propyl, 4-methylphenyl, 4-methoxyphenyl Reaction condition: e: $\mathrm{NaNO}_{2}, \mathrm{HCl}$, 0-5 $\mathrm{C}$; : $\mathrm{CH}_{3} \mathrm{COCH}_{2} \mathrm{COOC}_{2} \mathrm{H}_{5}, \mathrm{CH}_{3} \mathrm{COONa}$; : $\mathrm{NH}_{2} \mathrm{OH}, \mathrm{CH}_{3} \mathrm{COONa}$. 


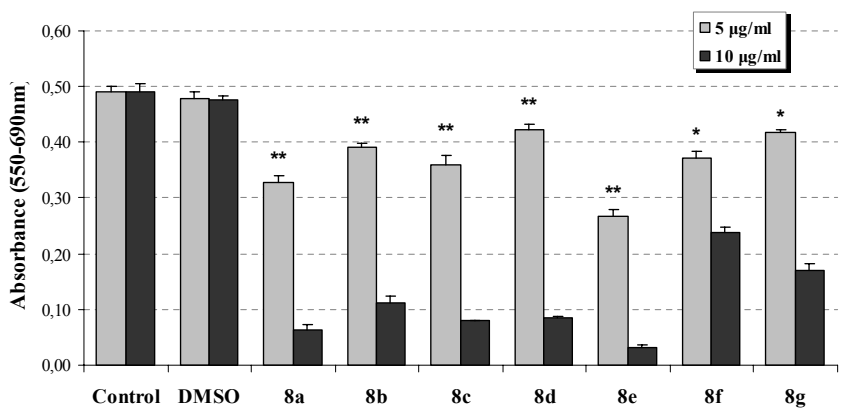

FIGURE 1. Cytotoxic activity profile of the compounds using MTT assay $\left({ }^{*} p<0.05,{ }^{* *} p<0.001\right)$

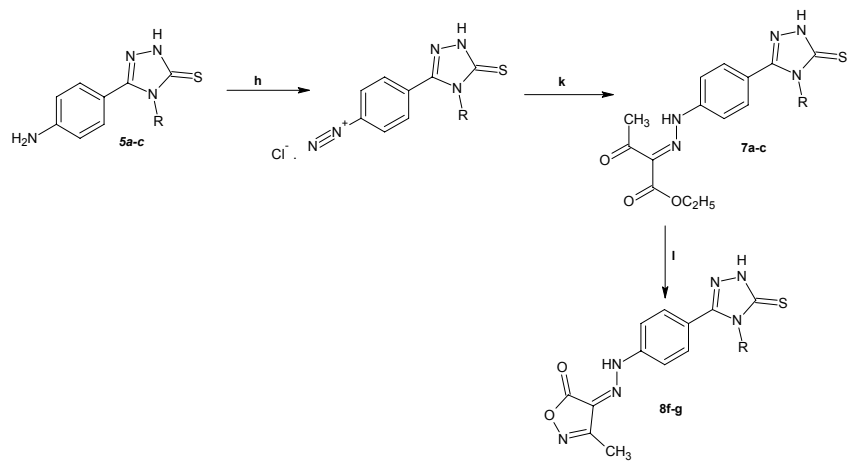

SCHEME 3. Synthesis of 5-isoxazolone derivatives (8f-g)

$\mathrm{R}=$ methyl, ethyl, propyl, 4-methylphenyl, 4-methoxyphenyl

Reaction condition: h: $\mathrm{NaNO}_{2}, \mathrm{HCl}, 0-5^{\circ} \mathrm{C}$; $\mathrm{k}: \mathrm{CH}_{3} \mathrm{COCH}_{2} \mathrm{COOC}_{2} \mathrm{H}_{5}, \mathrm{CH}_{3} \mathrm{COONa}$; I: $\mathrm{NH}_{2} \mathrm{OH}, \mathrm{CH}_{3} \mathrm{COONa}$

The cyclization of 5-isoxazolone ring for compounds 8a-g was evidenced by its ${ }^{1} \mathrm{H}-\mathrm{NMR}$ and IR spectra. The ${ }^{1} \mathrm{H}-\mathrm{NMR}$ spectra of compounds 8a-g showed a sharp singlet at 2.27-2.31 ppm due to methyl protons of 5-isoxazolones. Also, the IR spectrum of compounds $8 \mathbf{a - g}$, isoxazolone $\mathrm{C}=\mathrm{O}$ stretching bands were determined at $1716-1720 \mathrm{~cm}^{-1}$. On the other hand, the hydrazone N-H streching bands were observed at 3032-3207 $\mathrm{cm}^{-1}$. Hydrazone and isoxazolone $\mathrm{C}=\mathrm{N}$ bands were detected at $1506-1553 \mathrm{~cm}^{-1}$.

The ${ }^{1} \mathrm{H}-\mathrm{NMR}$ spectra of compounds containing 1,3,4-thiadiazole moiety (8a-g) displayed NH resonances at 7.98-10.52 ppm assigned for secondary amine $\mathrm{NH}$ protons (34). The ${ }^{1} \mathrm{H}-\mathrm{NMR}$ spectra of compounds containing 1,2,4-triazole-3-thione, displayed triazole $\mathrm{NH}$ resonances at 13.95-14.09 ppm. All of compounds displayed hydrazone $\mathrm{NH}$ resonances at 12.72-12.91 ppm (35).

Cytotoxicity of the seven compounds were evaluated by using HEK293 cell line according to procedures of MTT [3-(4,5-dimethylthiazole-2-yl)-2,5-diphenyltetrazolium bromide] assay. All the compounds were dissolved in dimethylsulfoxide $(2.5 \%)$ and no cytotoxic effect was observed when compared with the control group. Two different concenterations (5.0 and $10.0 \mathrm{mg} / \mathrm{mL}$ ) were used. Cell viability and cytotoxic activity profile of the compounds were analyzed using the MTT assay.

Cytotoxic activity results were presented in Figures 1, 2 and 3. Among the tested compounds, two compounds showed 10-

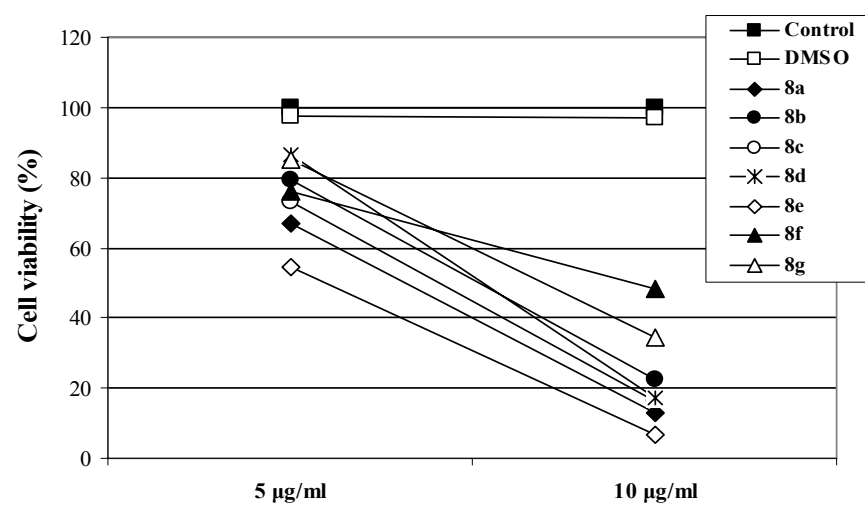

FIGURE 2. Cell viability (\%) of compounds $8 \mathrm{a}-\mathrm{g}$

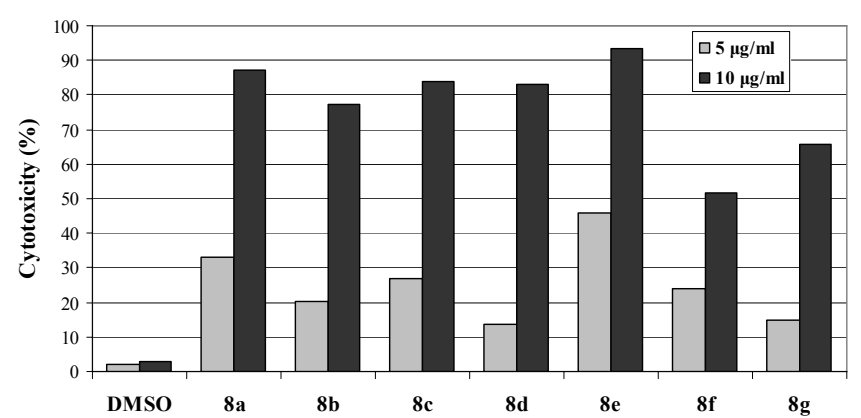

FIGURE 3. Cytotoxic activity (\%) of compounds $8 \mathrm{a}-\mathrm{g}$

$20 \%$, four compounds showed $20-35 \%$ and one compound showed $45.72 \%$ cytotoxic activity. The highest inhibitions were confirmed as $45.72 \%$ for the compound 3-methyl-4-[2-(4-\{5-[(4methoxyphenyl)amino]-1,3,4-thiadiazol-2-yl\}phenyl)hydrazinylidene]isoxazol-5(4H)-one (8e) and $33.07 \%$ for the compound 3-methyl-4-[2-(4-\{5-[(4-methylphenyl)amino]-1,3,4-thiadiazol-2-yl\}phenyl)hydrazinylidene]isoxazol-5(4H)-one (8a). As these values were under $50 \%$ we can conclude that neither compound displayed cytotoxic activity at $5.0 \mu \mathrm{g} / \mathrm{mL}$ concentration. All the compounds were tested at concentrations of 5.0 and $10.0 \mathrm{mg} / \mathrm{mL}$ and they showed a dose-related effect at these concentrations ( $p<0.001$ for compound $8 a, 8 b, 8 c, 8 d$ and 8e; $\mathrm{p}<0.05$ for compound $8 \mathrm{f}$ and $8 \mathrm{~g}$ ).

\section{CONClusion}

A series of 5-isoxazolone derivatives have been synthesized starting from substituted 1,3,4-thiadiazoles and 1,2,4-triazole3 -thiones and screened for their cytotoxic activities against HEK293 cell line. The cytotoxicity screening indicated that among the tested compounds, 5-isoxazolone derivatives carrying 5-methyl/methoxyphenylamino-1,3,4-thiadiazole ring (compound 8a and 8e) exhibited noteworthy activity.

\section{ACKNOWLEDGEMENT}

This study was supported by the Marmara University Scientific Research Committee (Project No:SAG-C-YLP-040609-0144, 2010 ). 


\section{Sübstitüe izoksazolon türevlerinin sentezi ve sitotoksik aktivitelerinin değerlendirilmesi}

ÖZET: Sübstitüe 1,3,4-tiyadiazol ve 1,2,4-triazol-3-tiyon bileşiklerinden hareketle bazı izoksazolon türevi bileşikler sentezlenmiştir. Araştırmanın birinci bölümünde, 2-(4-aminofenil)-5-alkil/arilamino-1,3,4-tiyadiazol yapısındaki bileşikler ve 5-(4-aminofenil)-4-sübstitüe-2,4-dihidro-3H-1,2,4-triazol-3 tiyon yapısındaki bileşikler, etil 4-amino benzoat'tan hareketle literatür yöntemine göre sentezlenmiştir. Araştırmanın ikinci bölümünde triazol ve tiyadiazollerin etil asetoasetat ile kenetlenmeleriyle oluşan ürünlerin hidroksilamin hidroklorür ve sodyum asetatla, etanollü ortamda siklizasyonu sonucunda 3-metil-4-\{2-[4-(5-alkil/arilamino-1,3,4-tiyadiazol-2-il)fenil]hidraziniliden\}-izoksazol5(4H)-on ve 3-metil-4-\{2-[4-alkil/aril-5-tiyokso-4,5-dihidro-1H-1,2,4-triazol-3-il)fenil]hidraziniliden\}izoksazol-5(4H)on' lar elde edilmiştir. Sentezlenen izoksazolon bileşiklerinin yapıları elementel analiz (C,H,N,S), UV, IR, 1H-NMR ve kütle spektroskopik yöntemleri kullanılarak aydınlatılmıştır. Sentezlenen bileşiklerin sitotoksik aktiviteleri HEK293 hücre hattı kullanılarak MTT [3-(4,5-dimetiltiyazol-2-il)-2,5-difeniltetrazolyum bromür] testi ile tayin edilmiştir. En yüksek düzeyde sitotoksik etki gösteren bileşikler; \% 45.72 ile 3-metil-4-[2-(4-\{5-[(4-metoksifenil)amino]-1,3,4-tiyadiazol2-il\}fenil)hidraziniliden] isoksazol-5(4H)-on (8e) ve \% 33.07 ile 3-metil-4-[2-(4-\{5-[(4-metilfenil)amino]-1,3,4-tiyadiazol2-il\}fenil)hidraziniliden]isoksazol-5(4H)-on (8a) bileşikleridir.

ANAHTAR KELIMELER: 1,2,4-Triazol-3-tiyon, 1,3,4-tiyadiazol, izoksazolon, hidrazon, sitotoksik aktivite

\section{REFERENCES}

1. Flores AFC, Zanatta N, Rosa A, Brondani S, Martins MAP. Synthesis of hydroxypyrazoles and 1-methyl-3isoxazolones via haloform reactions. Tetrahedron Lett 2002; 43: 5005-5008.

2. Ishioka $T$, Tanatani $A$, Nagasawa $K$, Hashimoto $Y$. AntiAndrogens with full antagonistic activity toward human prostate tumor LNCaP cells with mutated androgen receptor. Bioorg Med Chem 2003; 13: 2655-2658.

3. Demers J, Hageman W, Johnson S, Klaubert D, Look $\mathrm{R}$, Moore J. Selective inhibitors of protein kinase $\mathrm{C}$ in a model of graft-vs-host disease. Bioorg Med Chem 1994; 4: 2451-2456.

4. Ergenç N, Çapan G, Ötük G. New arylhydrazono-5(4H)isoxazolone derivatives as possible antibacterial and anticonvulsant agents. Pharmazie 1993; 48: 780-782.

5. Tamura N, Matsushta $Y$, Iwana $T$, Harada S, Kishimoto $S$, Itoh K. Synthesis and biological activity of (S)-2-amino-3-(2,5-dhydro-5-oxo-4-isoxazolyl)propanoic acid (TAN-950 A) derivatives. Chem Pharm Bull 1991; 39: 1199-1212.

6. Tamura N, Iwama $T$, Itoh $K$. Synthesis and glutamateagonistic activity of (S)-2-amino-3-(2,5-dihydro-5-oxo3-isoxazolyl)-propanoic acid derivatives. Chem Pharm Bull 1992; 40: 381-386.

7. Oral B. Synthesis and biological activities of hydrazones derived from five membered heterocylic rings. M U Institute of Health Sciences PhD thesis 2007; İstanbul.

8. Chande MS, Verma RS, Barve PA, Khanwelkar RR, Vaidya RB, Ajaikumar KB. Facile synthesis of active antitubercular, cytotoxic and antibacterial agents: A Michael addition approach. Eur J Med Chem 2005; 40: 1143-1148.

9. Zhang J, Didierlaurent S, Fortin M, Lefrancois D, Uridat E, Vevert JP. Nonpeptide endothelin antagonists: from lower affinity pyrazol-5-ols to higher affinity pyrazole-5carboxylic acids. Bioorg Med Chem Lett 2000; 10: 13511355.

10. Selwood DL, Brummell DG, Glen RC, Goggin MC, Reynolds K, Tatlock MA, Wishart G. Solution-phase parallel synthesis of 5-carboxamido 1-benzyl-3-(3-
dimethylaminopropyloxy)-1H-pyrazoles as activators of soluble guanylate cyclase with improved oral bioavailability. Bioorg Med Chem Lett 2001; 11: 1089-1092.

11. Abbiati G, Beccalli EG, Broggini G, Zoni CA. Valuable heterocyclic ring transformation: from isoxazolin-5(2H)ones to quinolines. Tetrahedron 2003; 59: 9887-9893.

12. Frydenvang $K$, Brehm $L$, Di Biase R, Falch E. 2-Ethyl3-(3-piridinil)-5(2H)-isoxazolone. Acta Cryst 1996; 52: 2102-2105.

13. Kömürcü ŞG, Rollas $S, Y_{1} l$ maz N, Çevikbaş A. Synthesis of 3-methyl-4-[(2,4-dihydro-4-substituted-3H-1,2,4-triazole-3-thione-5-yl)phenylhydrazono]-5-isoxazolone and evaluation of their antimicrobial activities. Drug Metab Drug Inter 1995; 12: 161-168.

14. Khan I, Sajid A, Hameed $S$, Rama NH, Hussain MT, Wadood A, Udin R, Ul-Haq Z, Khan A, Ali S, Choudhary MI. Synthesis, antioxidant activities and urease inhibition of some new 1,2,4-triazole and 1,3,4-thiadiazole derivatives. Eur J Med Chem 2010; 45: 5200-5207.

15. Holla B S, Veerenda B, Shivananda K, Boja P. Synthesis characterization and anticancer activity studies on some Mannich bases derived from 1,2,4-triazoles. Eur J Med Chem 2003; 38: 759-767.

16. Abdel-Megeed AM, Abdel-Rahman HM, Alkaramany GE, El-Gendy MA. Design, synthesis and molecular modeling study of acylated 1,2,4-triazole-3-acetates with potential anti-inflammatory activity. Eur J Med Chem 2009; 44: 117-123.

17. Kumar H, Javed SA, Khan SA, Amir M. 1,3,4-Oxadiazole/thiadiazole and 1,2,4-triazole derivatives of biphenyl-4-yloxy acetic acid: Synthesis and preliminary evaluation of biological properties. Eur J Med Chem 2008; 43: 2688-2698.

18. Kaplancikli ZA, Turan-Zitouni G, Ozdemir A, Revial G. New triazole and triazolothiadiazine derivatives as possible antimicrobial agents. Eur J Med Chem 2008; 43: 155159.

19. Küçükgüzel İ, Küçükgüzel $G$, Rollas $S$, Otük-Saniş $G$, Ozdemir O, Bayrak I, Altuğ T, Stables, JP. Synthesis of some 3-(arylalkylthio)-4-alkyl/aryl-5-(4-aminophenyl)- 
$4 \mathrm{H}-1,2,4$-triazole derivatives and their anticonvulsant activity. Farmaco 2004; 59: 893-901.

20. Küçükgüzel İ, Tatar E, Küçükgüzel ŞG, Rollas $S$, Clercq ED. Synthesis of some novel thiourea derivatives obtained from 5-[(4-aminophenoxy)methyl]-4- alkyl/aryl2,4-dihydro-3H-1,2,4-triazole-3-thiones and evaluation as antiviral/anti- HIV and antituberculosis agents. Eur J Med Chem 2008; 43: 381-392.

21. Tozkoparan B, Küpeli E, Yeşilada E, Ertan M. Preparation of 5-aryl-3-alkylthio-1,2,4-triazoles and corresponding sulfones with antiinflammatory-analgesic activity. Bioorg Med Chem 2007; 15: 1808-1814.

22. Doğan $H N$, Duran $A$, Rollas $S$. Synthesis and preliminary anticancer activity of new $1 \mathrm{H}-4,5$-dihydro-3-(3-hydroxy2-naphthyl)-4-substituted-1,2,4-triazole-3-thiones. Indian J Chem Sec B 2005; 44: 2301-2307.

23. Ashok M, Holla BS, Boja P. Convenient one pot synthesis and antimicrobial evaluation of some new Mannich bases carrying 4-methylthiobenzyl moiety. Eur J Med Chem 2007; 42: 1095-1101.

24. Rollas $S$, Kalyoncuoğlu N, Sür-Altıner D, Yeğenoğlu $Y$. 5-(4-Aminophenyl)-4-substituted-2,4-dihydro-3H-1,2,4triazole-3-thiones:Synthesis and antibacterial and antifungal activities. Pharmazie 1993; 48: 308-309.

25. Schenone $S$, Brullo $C$, Bruno $O$, Bondavalli $F$, Ranise A, Flippelli W, Rinaldi B, Capuano A, Falcone G. New 1,3,4-thiadiazole derivatives endowed with analgesic and anti-inflammatory activities. Bioorg Med Chem 2006; 14: 1698-1705.

26. Rzeski W, Matysiak J, Szerszén MK. Anticancer, neuroprotective activities and computational studies of 2-amino-1,3,4-thiadiazole based compound. Bioorg Med Chem 2005; 15: 3201-3207.

27. Mavrova AT, Wesselinova $D$, Tsenov $Y A$, Denkova $P$.
Synthesis, cytotoxicity and effects of some 1,2,4-triazole and 1,3,4-thiadiazole derivatives on inmunocompetent cells. Eur J Med Chem 2009; 44: 63-69.

28. Kokyan Ş. Synthesis and evaluation of cytotoxic activities of some substituted isoxazolone derivatives. MU Institute of Health Sciences MSc thesis 2010; İstanbul.

29. Mossman T. Rapid colorimetric assay for cellular growth and survival: Application to proliferation and cytotoxicity assays. J Immunol Methods 1983; 65: 55-63.

30. Beekman AC, Barentsen AR, Woerdenbag HJ, Uden WV, Pras N. Stereochemistry-dependent cytotoxicity of some artemisinin derivatives. J Nat Prod 1997; 60: 325-330.

31. Srivastava V, Negi AS, Kumar JK, Faridi U, Sisodia BS, Darokar MP, Luqman S, Khanuja SPS. Synthesis of 1 -( $3^{\prime}, 4^{\prime}, 5^{\prime}$-trimethoxy)phenyl naptho[2,1b]furan as a novel anticancer agent. Bioorg Med Chem 2006; 16: 911914.

32. Karakuş $S$, Rollas $S$. Synthesis and antituberculosis activity of new N-phenyl-N'-[4-(5 alkyl/ arylamino-1,3,4thiadiazole-2-yl)phenyl]thioureas. Farmaco 2002; 57: 577-581.

33. Solak N, Rollas $S$. Synthesis and antituberculosis activity of 2-(aryl/alkylamino)-5-(4-aminophenyl)-1,3,4-thiadiazoles and their schiff bases. Arkivoc 2006; 12: 173-181.

34. Karakuş $S$, Çoruh U, Barlas-Durgun B, Vázquez-López EM, Özbaş-Turan S, Akbuğa J, Rollas S. Synthesis and cytotoxic activity of some 1,2,4-triazoline-3-thione and 2,5-disubstituted- 1,3,4-thiadiazole derivatives. Marmara Pharm J 2010; 14: 84-90.

35. Küçükgüzel G, Rollas S, Erdeniz H, Kiraz M, Ekinci CA, Vidin A. Synthesis, characterization and pharmacological properties of some 4-arylhydrazono-2-pyrazoline-5one derivatives obtained from heterocyclic amines. Eur J Med Chem 2000; 35: 761-771. 